\author{
S. Rao Mallampati MD, Stephen P. Gatt MD, \\ Laverne D. Gugino, PH D, MD, \\ Sukumar P. Desai MD, Barbara Waraksa CRNA, \\ Dubravka Freiberger MD, Philip L. Liu MD
}

\section{A clinical sign to predict difficult tracheal intubation: a prospective study}

It has been suggested that the size of the base of the tongue is an important factor determining the degree of difficulty of direct laryngoscopy. A relatively simple grading system which involves preoperative ability to visualize the faucial pillars, soft palate and base of uvula was designed as a means of predicting the degree of difficulty in laryngeal exposure. The system was evaluated in 210 patients. The degree of difficulty in visualizing these three structures was an accurate predictor of diffculty with direct laryngoscopy $(p<0.001)$.

\section{Key words}

INTUBATION, ENDOTRACHEAL: complications, difficult intubation; ANAESTHETIC TECHNIQUES: laryngoscopy.

Unexpected difficulties in endotracheal intubation can be a significant aetiologic factor in morbidity and mortality in clinical practice. However, it is not always possible to predict from usual clinical observations in which patient orotracheal intubation may prove difficult. This is particularly true in individuals who offer significant difficulty for orotracheal intubation despite unremarkable orofacial anatomic features.

The principal author of this paper (S.R.M.) previously proposed a "clinical sign to predict difficult tracheal intubation (hypothesis)." The clinical sign is the concealment of faucial pillars (palatoglossal and palatopharyngeal arches) and

From the Department of Anaesthesia, Brigham and Women's Hospital, Harvard Medical School, Boston, Massachusetts.

Address correspondence to: Dr. S. Rao Mallampati, Brigham and Women's Hospital, 75 Francis Street, Boston, Massachusetts 02115. uvula by the base (posterior part) of the tongue, when the latter is maximally protruded in a seated patient. The anatomic basis of the sign is explained by the hypothesis as follows. If the base of the tongue is disproportionately large, it overshadows the larynx, rendering the exposure of the latter by direct laryngoscopy poor or difficult. A large tongue is also likely to mask the visibility of the faucial pillars and the posterior part of the soft palate where the uvula is an easily recognizable landmark. Since it is not possible to determine the volume or size of the base of tongue relative to the capacity of the oropharyngeal cavity, it is also logical to infer that the base of the tongue is disproportionately large when it is able to mask the visibility of the faucial pillars and uvula.

This clinical sign was evaluated in a prospective study which was designed and conducted to ascertain the sign's usefulness in predicting the ease or difficulty of orothacheal intubation in clinical practice.

\section{Methods}

The study was approved by the "Human Subjects for Research Studies Committee" of our institution.

Two hundred and ten adult surgical patients (47 men and 163 women; Table I), American Society of Anesthesiologists (ASA) physical status 1 or 2, who required general endotracheal anaesthesia were included in the study. One hundred and ninety nine patients had full dentition. Three patients had no maxillary teeth. Eight patients were edentulous. Twelve patients had prominent maxillary incisors resulting in "overbite." Patients were chosen consecutively with few criteria for exclusion. All patients were in good general health. Patients with known cardiovascular or respiratory pathology were excluded.

Patients who were known to have any form of 
TABLE I Patient characteristics

\begin{tabular}{lllll}
\hline Total: 210 & $\begin{array}{l}\text { Age } \\
\text { (years) }\end{array}$ & $\begin{array}{l}\text { Height } \\
(\mathrm{cm})\end{array}$ & $\begin{array}{l}\text { Weight } \\
(\mathrm{kg})\end{array}$ \\
\hline Male: 47 & Range & $18-82$ & $150-208$ & $45-145$ \\
Female: 163 & Mean & 39.32 & 163.81 & 70.33 \\
\hline
\end{tabular}

arthritis but were free from cardiovascular or respiratory disease were included in the study. Five patients had rheumatoid arthritis and one had osteoarthritis. All six patients had multiple joint involvement but none was incapacitated. Involvement of temporomandibular and cervical joints, if any, was not found to be clinically significant. Four patients who were obese (mean weight: $136 \mathrm{~kg}$; range $130-145 \mathrm{~kg}$ ) and underwent gastroplasty for weight control were also included.

Preoperative assessment also included neck mobility. Two hundred and six patients were found to have unrestricted mobility of head and neck. Movements of neck were found to be moderately restricted in four patients. None of the patients had known upper airway pathology.

Twenty-two individuals (6 staff physicians, ten resident physicians, and six nurse anaesthetists) who each had a minimum of two years' experience in clinical anaesthesia carried out airway assessment and laryngoscopy grading according to the protocol. The patient's airway was assessed at the time of the preoperative interview by the individual scheduled to administer anaesthesia. Induction and laryngoscopy grading were subsequently carried out by the same individual. Visibility of pharyngeal structures (faucial pillars, soft palate, and base of uvula) was noted by instructing the patient to open his/her mouth and protrude the tongue maximally while in the sitting posture. Each patient was directed to perform the manoeuvre at least twice to minimize the chances for erroneous observation.

Patients were divided into three classes:

Class 1 Faucial pillars, soft palate and uvula could be visualized.

Class 2 Faucial pillars and soft palate could be visualized, but uvula was masked by the base of the tongue.

Class 3 Only soft palate could be visualized.

Figure 1 illustrates a patient whose faucial pillars and uvula can be fully visualized. Figure 2 shows a

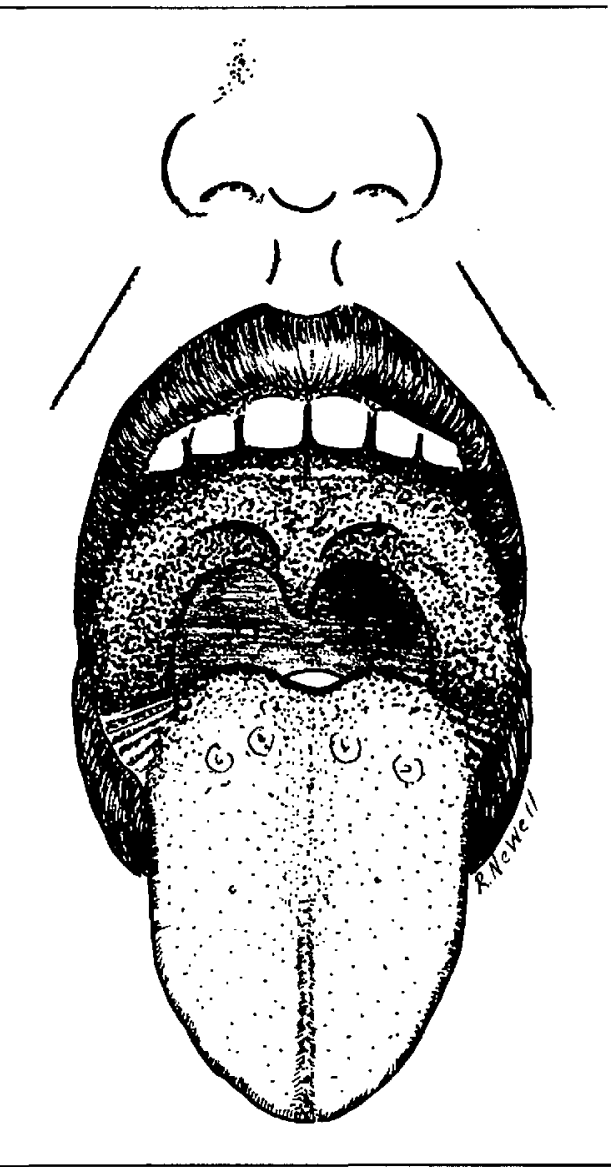

FIGURE 1 Illustration of patient in whom faucial pillars, soft palate and uvula are visible.

patient whose faucial pillars and uvula are concealed by the base of the tongue even when the latter is maximally protruded.

Premidication consisted of either diazepam $(10 \mathrm{mg})$ orally or morphine $(5-10 \mathrm{mg})$ and atropine $(0.4 \mathrm{mg})$ intramuscularly given 1.5 hours before induction of anaesthesia. In the operating room, a standard induction and intubation protocol was used for all patients as follows:

Position: Supine with head in the "sniffing position" (flexion of the cervical spine and extension of the head at the atlanto-occipital joint) so that the patient's face was at the level of the anesthetist's xiphoid process. 


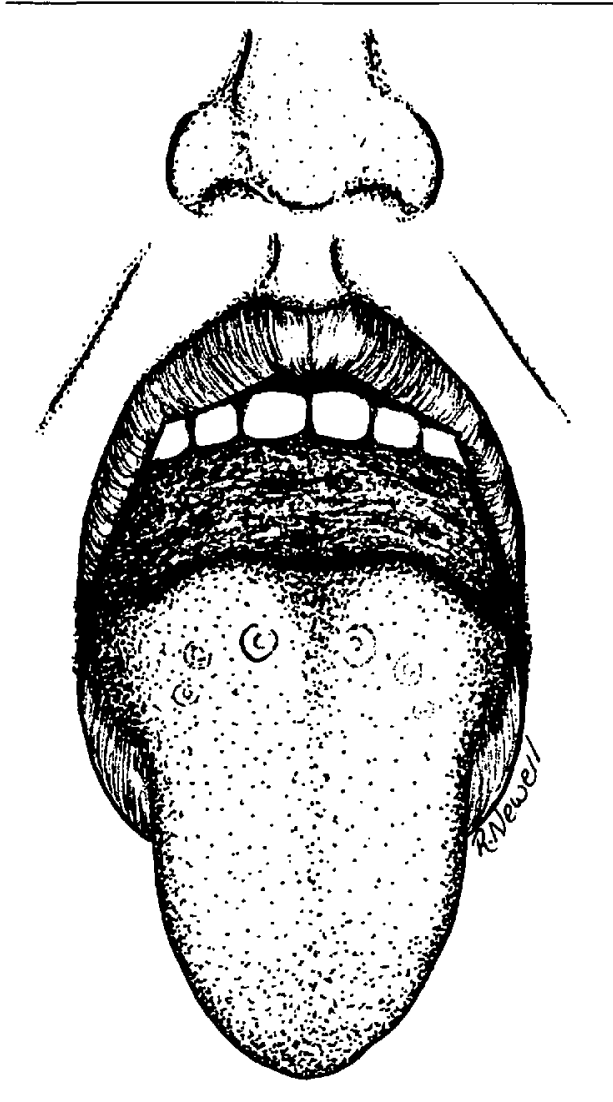

FIGURE 2 Illustration of patient where none of the three pharyngeal structures are visible.

Induction: Thiopentone $5 \mathrm{mg} \cdot \mathrm{kg}^{-1}$ (or more if necessary) until the loss of eyelash reflex was achieved.

Muscle relaxant: Succinylcholine $1 \mathrm{mg} \cdot \mathrm{kg}^{-1}$ intravenously to facilitate endotracheal intubation (precurarization was not used).

Laryngoscopy: After the disappearance of fasciculations in the face and neck muscles, laryngoscopy was performed using a \# 3 Macintosh blade.

For the purpose of this study, difficult orotracheal intubation was defined as less than adequate exposure of the glottis by direct laryngoscopy. The extent of exposure of the glottis is expressed on a scale of 1 to 4 as follows:
Grade 1 Glottis (including anterior and posterior commissures) could be fully exposed.

Grade 2 Glottis could be partly exposed (anterior commissure not visualized).

Grade 3 Glottis could not be exposed (corniculate cartilages only could be visualized).

Grade 4 Glottis including corniculate cartilages could not be exposed.

Grades 1 and 2 were considered "adequate exposure" and grades 3 and 4 "inadequate exposure."

The statistical significance of the data was analyzed using the Chi-square test. Statistical significance was assumed when $p<0.05$.

\section{Results}

Table II shows the correlation between the visibility of pharyngeal structures (faucial pillars, soft palate and uvula) and difficulty with intubation. A significant correlation exists between the ability to visualize pharyngeal structures and the ease of laryngoscopy (Chi-square: $\mathrm{p}<0.001$ ).

In Class 1 (155 patients) all three pharyngeal structures (faucial pillars, soft palate and uvula) were visible. In all these patients laryngoscopic visualization proved relatively easy, i.e., either grade 1 ( 80.6 per cent) or grade 2 (19.3 per cent). In this group there were no difficult visualizations (grades 3 or 4 ). In Class 2 ( 40 patients) whose uvula was masked by the base of the tongue but faucial pillars and soft palate were visible, laryngoscopy proved to be easy (grade 1 or 2 ) in 26 patients (65 per cent) and difficult (grades 3 or 4 ) in 14 (35 per cent). In Class 3 (15 patients) only part of the soft palate was visible, the faucial pillars and uvula being masked by tongue as depicted in Figure 2. Of the 15 patients, there were no full (grade 1) visualizations and only one ( 6.6 per cent) easy exposure. All the other patients had either grade 3 (60 per cent) or grade 4 laryngoscopy (33.3 per cent).

Exposure of the glottis was adequate (grade 1 or 2) in all eight edentulous patients as well as in the three patients who had no maxillary teeth. In the 12 patients with overbite of teeth, exposure of the glottis was adequate in nine and inadequate (grade 3 or 4 ) in three. Of the four patients with moderately restricted mobility of neck, two had adequate glottic exposure and two inadequate glottic exposure. All 
TABLE II Correlation between visibility of faucial pillars, soft palate and uvula, and exposure of glottis by direct laryngoscopy

\begin{tabular}{lllll}
\hline Visibility of & \multicolumn{3}{l}{ Laryngoscopy grade } & \multicolumn{2}{l}{} & \\
\cline { 2 - 5 } $\begin{array}{l}\text { structures } \\
\text { no. pts }(\%)\end{array}$ & $\begin{array}{l}\text { Grade I } \\
\text { no.pts }(\%)\end{array}$ & $\begin{array}{l}\text { Grade } 2 \\
\text { ro.pts }(\%)\end{array}$ & $\begin{array}{l}\text { Grade } 3 \\
\text { no.pts }(\%)\end{array}$ & $\begin{array}{l}\text { Grade 4 } \\
\text { no.pts (\%) }\end{array}$ \\
\hline $\begin{array}{l}\text { Class 1 } \\
155(73.8 \%)\end{array}$ & $125(59.5 \%)$ & $30(14.3 \%)$ & - & - \\
$\begin{array}{l}\text { Class 2 } \\
40(19 \%)\end{array}$ & $12(5.7 \%)$ & $14(6.7 \%)$ & $10(4.7 \%)$ & $4(1.9 \%)$ \\
$\begin{array}{l}\text { Class 3 } \\
15(7.14 \%)\end{array}$ & - & $1(0.5 \%)$ & $9(4.3 \%)$ & $5(2.4 \%)$ \\
\hline
\end{tabular}

Class 1: Faucial pillars, soft palate and uvula could be visualized. Class 2: Faucial pillars and soft palate could be visualized, but uvula was masked by the base of the tongue. Class 3: Only soft palate could be visualized. Grade 1: Glottis (including anterior and posterior commissures) could be fully exposed. Grade 2: Glottis could be partly exposed (anterior commissure not visualized). Grade 3: Glortis could not be exposed (corniculate cartilages only could be visualized). Grade 4: Glottis including coniculate cartilages could not be exposed.

four patients who underwent gastroplasty for obesity had adequate glottic exposure.

\section{Discussion}

The significance of difficult or failed tracheal intubation following induction is well-recognized as a cause of morbidity and mortality in anaesthetic practice. Nevertheless, the need to predict a potentially difficult tracheal intubation has received little attention.

Many anatomic characteristics have been suggested as useful in assessing anticipated difficulty of tracheal intubation. Cass, James and Lines ${ }^{2}$ described five cases of difficult direct laryngoscopy and analyzed the anatomic features that make visualization of the glottis difficult. These include: a short muscular neck with a full set of teeth; a receding mandible with obtuse mandibular angles; protruding maxillary incisor teeth because of relative overgrowth of premaxilla; poor mobility of the mandible associated with temporomandibular arthritis or trismus; a long high arched palate associated with a long narrow mouth; and increased alveolar-mental distance necessitating wider opening of the mouth.

White and Kander ${ }^{3}$ studied radiographs of the mandible, upper jaw and cervical spine in thirteen patients in whom direct laryngoscopy was difficult. They found that the posterior depth of the mandible (distance between the alveolus immediately behind third molar tooth and the lower border of mandible) was the most important factor in determining the ease of direct laryngoscopy. An increase in this measurement was thought to hinder displacement of the soft tissues by the laryngoscope blade.

Nichol and Zuck ${ }^{4}$ suggested that the atlantooccipital distance is a major anatomical factor that determines the ability to extend the head on the neck and exposure of larynx. Bannister and Macbeth ${ }^{5}$ stressed the importance of the position of the head and neck in direct laryngoscopy in order to achieve proper alignment of the axes of the mouth, pharynx and larynx. The ideal position for axes alignment and intubation can be achieved by raising the head, flexing the neck and extending the head at the atlanto-occipital joint (Gillespie). ${ }^{6}$ Failure to correctly position the head and neck and bring about alignment of oral, pharyngeal and laryngeal axes is one of the common errors in orotracheal intubation. ${ }^{7}$

A variety of pathological conditions could also render intubation difficult or impossible by altering or distorting the regional anatomy: congenital (micrognathia, Pierre Robin Syndrome, cleft lip and cleft palate, floppy epiglottis, large teeth to larynx distance), inflammatory (epiglottitis, Ludwig's angina, retropharyngeal abscess), arthritis of temporomandibular joint or cervical spine, ankylosing spondylitis of the cervical spine, burn contractures, fractures involving mandible, maxilla or cervical spine, endocrine causes (goiter, acromegaly) and neoplasia (pharyngeal and laryngeal tumors).

Unheralded difficult intubation is a risk to the 
patient and a challenge to the skill of the anaesthetist. In the absence of pathological conditions, while radiographic methods are time-consuming and cannot be routinely employed, prediction of the ease or difficulty of intubation rests on assessment of the aforementioned anatomical factors. Anatomical characteristics do offer clues to possible intubation difficulty, but have limitations because of their variability from one person to another and in the same person at different time periods in life. In order to be able to assess and describe with objectivity, we need to attempt a meaningful correlation of one anatomic variable with another in the same patient. Failure to do so would tend to make the same anatomic feature seem normal to one observer and abnormal to another. The goal, of course, is to develop a clinical method which is simple, easy to apply, and amenable to objectivity.

The method we have employed is based upon the hypothesis previously proposed by the principal author. It relates the size of the base of the tongue to the faucial pillars, soft palate and uvula. This method is especially important because the tongue is the largest single structure in the mouth affecting the accessibility of the laryngeal inlet to direct laryngoscopy. Since it is not possible to measure the size of the tongue relative to the capacity of the oropharyngeal cavity, we now have an indirect means of assessing the size of the base of the tongue.

Our study was conducted in a large group of patients (total: 210) of ASA physical status 1 or 2 . We necessarily excluded patients in ASA physical status 3 or 4 from the study since they might not tolerate the same doses of drugs as patients in physical status 1 or 2 . Although the sexes were unevenly distributed (47 males and 163 females), the total number in each sex is significantly large enough to enable us to extend the conclusions to the population at large.

Twenty two individuals who each had more than two years of experience in clinical anesthesia took part in carrying out preoperative assessment and laryngoscopy grading. Nevertheless, the principal author is the only individual who previously had experience with this new method of airway assessment. Since all the participants with the exception of the principal author were not previously aware of the usefulness or otherwise of this method, observer blindness was not thought to be warranted. The same individual who assessed the airway also performed the laryngoscopy, as per the protocol. Also, it was not practical to have the availability of a second anaesthetist to carry out airway assessment or laryngoscopy for a large group of patients as in this study.

Patients who had no teeth or had "overbite" of teeth or restricted mobility of the neck and obese patients warrant special mention. While it was generally easy to expose the glottis in all eight edentulous patients as well as in the three patients who had no maxillary teeth, three of the 12 patients with "overbite" of teeth had inadequate exposure. of the four patients with moderately restricted mobility of neck, two had adequate glottic exposure and two inadequate glottic exposure. "Overbite" of teeth and restricted mobility of the neck are thus certainly adverse factors. All four patients who underwent gastroplasty for obesity had adequate glottic exposure, consistent with the visibility of faucial pillars and uvula in the four patients

Our results are highly significant $(p<0.001)$ and support the hypothesis that difficult laryngeal visualization can be predicted in most cases by eliciting the visibility of faucial pillars and uvula. If all three pharyngeal structures (FP, SP, U) are visible, one can expect adequate exposure of larynx by direct laryngoscopy. On the other hand, if the faucial pillars and uvula are masked by the base of the tongue and only soft palate is visible, one should anticipate difficult endotracheal intubation secondary to inadequate exposure of larynx by direct laryngoscopy.

\section{Acknowledgements}

The authors acknowledge with gratitude the editorial advice of Benjamin G. Covino, Ph.D., M.D. and Leroy D. Vandam, M.D. We are also grateful to the members of the Department of Anesthesia, Brigham and Women's Hospital, for their cooperation; to Mr. Robert Newall for depiction of the clinical sign; and to Dianne Visconti for secretarial assistance.

\section{References}

1 Mallampati $S R$. Clinical sign to predict difficult tracheal intubation (hypothesis). Can Anaesth Soc J 1983; 30: 316-7. 
2 Cass NM, James NR, Lines V. Difficult direct laryn- Résumé goscopy complicating intubation for anaesthesia. Br Med J 1956; 1: 488-9.

Il a été suggéré que la grosseur de la base de la langue est un facteur important dans la détermination du degré de 3 White A, Kander PL. Anatomical factors in difficult difficulté de la laryngoscopie directe. Un système reladirect laryngoscopy. Br J Anaesth 1975: 47: 468-74.

4 Nichol HC, Zuck D. Difficult laryngoscopy - the tivement simple de classification impliquant la capacité "anterior" larynx and the atlanto-occipital gap. Bt J Anaesth 1983; 55: 141-3.

5 Bannister FB, MacBeth RG. Direct laryngoscopy and intubation. Lancet 1944; $2: 651-4$.

6 Gillespie NA. Endotracheal anesthesia. 2nd edition. Madison: University of Wisconsin Press, 1950. pré-opératoire de visualiser les piliers du voile du palais, le voile du palais et la base de la luette a été conģu afin de prédire le degré de la difficulté d'exposition du larynx. Ce système a été évalué chez 210 patients. On a trouvé que le degré de difficulté encourue lors de la visualisation de ces trois structures était un moyen précis pour prédire la 7 Salem MR, Mathrubhutham M, Bennet EJ. Difficult intubation. N Engl J Med 1976; 295: 879-81. 